\title{
Leishmaniose Canina em Brasília, DF: Uma Revisão da Literatura.
}

\author{
Leishmaniasis in dogs from Brasília, DF: a literature review.
}

Perro leishmaniasis en Brasilia, DF: comentarios sobre la literatura.

\author{
Tatyere Constâncio de SOUSA ${ }^{1}$ \\ Ariadine Kelly Pereira Rodrigues FRANCISCO ${ }^{22}$ \\ Isabele Barbieri dos SANTOS ${ }^{3}$
}

RESUMO: A leishmaniose é uma doença infecto-parasitária que acomete seres humanos e animais, causada por protozoários do gênero Leishmania. As leishmanioses são importante problema de saúde pública em vários países e estão incluídas entre as seis endemias de maior relevância mundial. São classificadas em tegumentar (LT) cutânea e mucocutânea - e visceral $(\mathrm{LV})$. A transmissão ocorre através de picada de insetos flebotomíneos pertencentes aos gêneros Lutzomyia (Novo Mundo) e Phlebotomus(Velho Mundo). A Leishmaniose Tegumentar Americana (LTA) é uma zoonose distribuída no território brasileiro. Possui evolução crônica e acomete a pele e mucosas, isoladamente ou em associação, de seres humanos, cães, gatos e eqüinos. Nos cães, se caracteriza pela presença de lesões cutâneas ulceradas, recobertas por crostas, localizadas nas orelhas, bolsa escrotal, focinho e membros. A Leishmaniose visceral é uma zoonose que ocorre nas regiões subtropicais e tropicais, causada pela Leishmania (Leishmania) chagasi, no Novo e no Velho Mundo. No Brasil, a Leishmaniose Visceral Canina (LVC) coexiste com a doença humana e os cães são seu reservatório doméstico. A eutanásia do cão é criticada por isso enfrenta limitações. Apesar das ações de vigilância e controle de LV adotadas no DF, a doença permanece em áreas urbanas e rurais.

Palavras-chave:Doenças do cão, Leishmaniose, Zoonoses

ABSTRACT:Leishmaniasis is an infectious parasitic disease that affects humans and animals caused by protozoa of the genus Leishmania. Leishmaniasis are important public health problem in many countries and are included among the six endemic largest global relevance. They are classified into cutaneous (LT) cutaneous and mucocutaneous - and visceral (LV). Transmission occurs through the bite of sand flies insects belonging to the Lutzomyia (New World) and Phlebotomus (Old World).

\footnotetext{
${ }^{1}$ Estudante do curso de Saúde Coletiva da UnB, Faculdade de Ceilândia, e estagiária do Programa de Epidemiologia e Vigilância em Saúde (PEPIVS) - Fiocruz Brasília - Direb.E-mail:tatyere.bsb@ hotmail.com

${ }^{2}$ Estudante do curso de Saúde Coletiva da UnB, campus Darcy Ribeiro, e estagiária curricular do Programa de Epidemiologia e Vigilância em Saúde (PEPIVS) - Fiocruz Brasília - Direb.

${ }^{3}$ Médica veterinária, tecnologista em saúde pública. Programa de Epidemiologia e Vigilância em Saúde (PEPIVS). FiocruzBrasilia - Direb - Fundação Oswaldo Cruz. Tel: 556133294523 ou 556133294791 - email:isabelebarbieri@fiocruz.br; belebarbieri@yahoo.com.br
} 
The American Cutaneous Leishmaniasis (ACL) is a zoonotic disease widely distributed in Brazil. It has chronic course and affects the skin and mucous membranes, alone or in combination, to humans, dogs, cats and horses. In dogs, is characterized by the presence of ulcerated skin lesions, sometimes covered with crusts, usually unique, located on the ears, scrotum, muzzle and legs. Visceral leishmaniasis is a zoonotic disease that occurs in subtropical and tropical regions, caused by Leishmania (Leishmania) chagasi in the New and Old World. In Brazil, Canine Visceral

Leishmaniasis (CVL) coexists with human disease in all known outbreaks and often precedes it, and the dogs considered as the main domestic reservoir. Euthanasia of domestic reservoir is the most criticized Item LV control program and has limitations. Despite surveillance and LV control adopted in DF, the disease remains in urban and rural areas, which emphasizes the lack of studies which point to more effective measures to eliminate the vectors and reduce the prevalence of CVL.

KEY-WORDS: Dog's deseases; Leishamiasis; Zoonoses

RESUMEN: La leishmaniasis es una enfermedad parasitaria infecciosa que afecta a seres humanos y animales causadas por protozoos del género Leishmania. La leishmaniasis es importante problema de salud pública en muchos países y se incluyen entre los seis endémica mayor importancia a nivel mundial. Se clasifican en cutánea (LT) cutánea y mucocutánea - y visceral (LV). La transmisión se produce a través de la picadura de moscas de la arena insectos pertenecientes a la Lutzomyia (Nuevo Mundo) y Phlebotomus (Viejo Mundo). La Leishmaniasis Cutánea Americana (LCA) es una enfermedad zoonótica de amplia distribución en Brasil. Tiene un curso crónico y afecta a la piel y las membranas mucosas, solos o en combinación, para seres humanos, perros, gatos y caballos. En los perros, se caracteriza por la presencia de lesiones cutáneas ulceradas, a veces cubierto con costras, generalmente únicos, situados en los oídos, el escroto, hocico y piernas. La leishmaniasis visceral es una enfermedad zoonótica que se produce en las regiones subtropicales y tropicales, causada por Leishmania (Leishmania) chagasi en el Nuevo y Viejo Mundo. En Brasil, la leishmaniasis visceral canina (CVL) convive con la enfermedad humana en todos los brotes conocidos ya menudo precede, y los perros considerado como el principal reservorio interno. La eutanasia del depósito interno es el programa de control de LV artículo más criticado y tiene limitaciones. A pesar de la vigilancia y el control del LV adoptada en Brasilia (DF), la enfermedad sigue siendo en las zonas urbanas y rurales, con énfasis en la falta de estudios que apuntan a medidas más eficaces para eliminar los vectores y reducir la prevalencia de la CVL.

Palabras-clave: Enfermedadesdel perro, Leihsmaniasis, Zoonosis

\section{INTRODUÇÃO}

A leishmaniose é uma doença infecto-parasitária que acomete seres humanos e animais, causada por protozoários do gênero Leishmania. As leishmanioses são importante problema de saúde pública em vários países e estão incluídas entre as seis endemias de maior relevância mundial ${ }^{(1)}$. São ISSN 1982-8829 Tempus actas de saúde colet. Brasília, 9(3), 187-202, set, 2015. 
classificadas em tegumentar (LT) cutânea e mucocutânea - e visceral (LV). A transmissão ocorre através de picada de insetos flebotomíneos pertencentes aos gêneros Lutzomyia (Novo Mundo) e Phlebotomus (Velho Mundo).

A epidemiologia das leishmanioses é complexa e grande parte dos fatores ambientais que interferem na cadeia epidemiológica ainda não estão bem esclarecidos. Evidências sugerem que alguns parasitos, assim como seus vetores possam adaptar-se às mudanças ambientais, transformando padrões clássicos de transmissão ${ }^{(2),(3)}$. Considerada inicialmente uma zoonose silvestre, hoje os padrões epidemiológicos revelam normalmente uma endemia, principalmente em áreas onde a

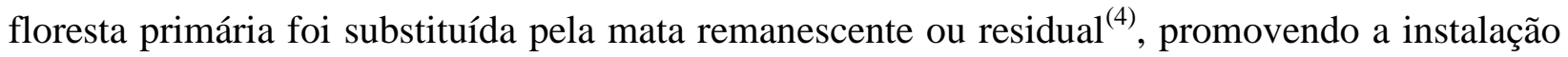
de focos em centros totalmente urbanizados com o envolvimento de animais domésticos no ciclo biológico $^{(3)}$.

O impacto das leishmanioses na saúde pública esteve extremante subestimado durante muitos anos e considera-se que 2 milhões de casos novos (1,5 milhões para LT e 500.000 para LV) ocorram anualmente, com uma estimativa de 12 milhões de pessoas infectadas atualmente em todo o mundo ${ }^{(1)}$.

Em diferentes focos de leishmaniose no Mundo, observa-se uma grande variedade de animais mamíferos incriminados como reservatório de diferentes espécies de Leishmania, pertencentes a distintos grupos taxonômicos, tanto silvestres quanto domésticos. Entre eles, destacamos o cão doméstico (Canis familiaris), principalmente pela proximidade com o homem. Este animal tem sido encontrado naturalmente infectado pelas seguintes espécies de Leishmania: L. (L.) infantum, L. (L.) donovani, L. (L.) tropica, L. (L.) chagasi, L. (V.) braziliensis, L. (V.) panamensis, L. (L.) mexicana, L. (V.) peruviana ${ }^{5}$. Pode ocorrer também infecção mista, ou seja, cães infectados com $L(V$.$) braziliensis e L(L$. $)$ chagasi ${ }^{6}$.No entanto, o cão doméstico tem importância epidemiológica como reservatórios domésticos no ciclo de transmissão somente de $L$. (L.) infantum e $L$. (L.) chagasi, devido a presença de cães infectados em áreas endêmicas de leishmaniose visceral e ao intenso parasitismo da pele sã dos cães ${ }^{7,8,9,10,11,12,13,14}$.

\section{Leishmaniose visceral}

A LV, também conhecida como calazar, é uma zoonose que ocorre nas regiões subtropicais e tropicais, causada por Leishmania (Leishmania) chagasi e Leishmania Leishmania infantum, no Novo e no Velho Mundo, respectivamente. Alguns autores acreditam que L. (L.) chagasi e $L$. infantum sejam a mesma espécie ${ }^{15,16}$. É endêmica em 62 países e cerca de 200 milhões de pessoas estão sob risco de adquirir a doença 1 . A maioria dos casos de LV ( $\geq 90 \%)$ está concentrada em países como Índia, Bangladesh, Nepal, Sudão, Etiópia e Brasil ${ }^{17},{ }^{18}$. Entretanto vem ocorrendo como doença emergente em humanos na Europa ${ }^{19}$ e em cães e gatos nos Estados Unidos ${ }^{20}$.

Os agentes etiológicos da LV são Leishmania (L.) infantum(região do Mediterrâneo, Ásia, China e norte da África), Leishmania (L.) donovani(Sudão, Índia, Bangladesh, Paquistão e Nepal), e ISSN 1982-8829 Tempus actas de saúde colet. Brasília, 9(3), 187-202, set, 2015. 
Leishmania (L.) chagasi(América Latina, exceto Chile e Uruguai) ${ }^{18}$.Alguns autores consideram a $L$. (L.) infantumsemelhante à $L$. (L.) chagasi, questionando sua classificação separadamente.

Entretanto, diferenças entre estasespécies quanto a estrutura molecular e sua antigenicidade foram evidenciadas, o que reforça a discriminação entre as espécies ${ }^{21,22}$.

Os vetores incriminados na transmissão da LV são flebotomíneos pertencentes ao gêneroLutzomyiano Novo Mundo e Phlebotomusno Velho Mundo ${ }^{23,24}$.Epidemiologicamente, existem dois tipos de LV que se diferem pelo modo da transmissão: LV zoonótica, transmitida do animal para o vetor e deste para o homem e LV antroponótica, transmitida do homem para o vetor e deste para o homem $^{18}$. A LV zoonótica é encontrada em áreas de transmissão de L (L.)chagasie de L (L.) infatum), enquanto que a LV antroponótica é encontrada em áreas de transmissão de L. (L.) donovani ${ }^{18,24}$.

No Brasil, a LV apresenta aspectos geográficos, climáticos e sociais diferenciados em função da sua ampla distribuição geográfica, envolvendo as regiões norte, nordeste, centro-oeste, sudeste e sul $^{25,26,27}$. De acordo com Grimaldi etal. $(1989)^{28}, 90 \%$ dos casos de LV notificados na América Latina são provenientes do Brasil, particularmente da região nordeste.

A LV no país apresentava eminentemente caráter rural, mas atualmente vem se expandindo para as áreas urbanas devido à degradação ambiental e falta de sanitização, associada à recente migração das populações rurais para os subúrbios das grandes cidades e a adaptação do inseto vetor às modificações ambientais ${ }^{3,29,30}$.

De acordo com o Ministério da Saúde $(2006)^{31}$, vem ocorrendo a peri-urbanização e urbanização da leishmaniose visceral, destacando-se os surtos ocorridos em Teresina (PI), Natal (RN), São Luíz (MA), Fortaleza (CE), Araçatuba (SP), Bauru (SP); Corumbá (MT), Campo Grande (MS), Palmas (TO); Brasília (DF), Rio de Janeiro (RJ), Belo Horizonte (MG); Monte Carlos (MG); Três Lagoas (MS); Rio Grande do Sul (Santa Maria e São Borja) $)^{25,32,33,34,35}$.

A espécie L. (L.) chagasi é o agente etiológico responsável pela LV no Brasil, sendo o flebotomíneo Lutzomyia longipalpis incriminado como principal vetor na sua transmissão. Entretanto, recentemente L. cruzi foi descrita no Estado do Mato Grosso do Sul exercendo esse papel ${ }^{31,36,37.38}$. Os reservatórios no ambiente silvestre são os roedores, as raposas (Dusicyon vetulus e Cerdocyon thous) e os marsupiais (Didelphis albiventris). Na área urbana o cão doméstico (Canis familiaris) é a principal fonte de infecção para o flebótomo.

O primeiro caso de LV canina no Brasil foi descrito em 193839, entretanto, Deane\&Deane, em $1955^{(7)}$, constataram a presença de formas amastigotas na pele de cães infectados, fato que facilitava a infestação pelos vetores, passando a incriminar o cão como um dos prováveis elos no ciclo de transmissão no ambiente doméstico. Desde então, a notificação de cães infectados em 
áreas endêmicas, associado ao aparecimento dos casos humanos, tem sugerido que o cão infectado possa manter e amplificar ciclos instalados no peridomicílio ${ }^{8,40,41,42}$. No Brasil, por essa razão, os órgãos sanitários competentes passaram a adotar medidas para interromper este ciclo de transmissão, por meio da detecção e eliminação dos cães infectados ${ }^{(31)}$.

A enzootia canina tem precedido a ocorrência de casos humanos e a infecção destes tem sido mais prevalente do que nos humanos ${ }^{3,8,38,42,43}$. A LV humana e canina coexistem de forma endêmica em bairros localizados no município de Bauru - São Paulo e no Município do Rio de Janeiro, no entorno do maciço da Pedra Branca (Realengo, Bangú, Senador Camará e Campo Grande) e na região litorânea (Barra de Guaratiba) ${ }^{8,44}$. A LVC é considerada mais importante que a LV humana do ponto de vista epidemiológico, pois, além de ter maior prevalência, apresenta grande contingente de animais assintomáticos ${ }^{45}$. O quadro clínico da LVC é variável, apresentando-se desde aparente estado sadio a quadros severos de caquexia associada a outras complicações $^{8,24}$. A doença também é bastante variável, podendo ser de uma forma aguda e grave, levando o animal a óbito em poucas semanas, evoluir de uma forma lenta, que pode durar anos, acompanhados ou não de sintomas que pode evoluir para uma forma grave ${ }^{8,46}$.

Classicamente, a LVC se caracteriza por caquexia progressiva, anemia, febre intermitente, linfadenomegalia generalizada, sinais oculares (conjuntivite, ceratoconjuntivite, uveite) e lesões cutâneas (dermatiteexfoliativa, úlceras e alopecia) e onicogrifose. Outros sinais tais como: colite, ceratoconjuntivite e insuficiência renal podem estar presentes. Formas atípicas também têm sido descritas $^{47}$. Acredita-se que o amplo espectro da apresentação clínica dependa da fase da doença e da condição imunológica do cão, o que pode dificultar o diagnóstico clínico. Outras doenças com manifestações semelhantes também podem contribuir para dificultar o diagnóstico.

Mancianti et al. (1988)48 sugerem que cães com LV sejam classificados de acordo com sinais clínicos da doença em: assintomáticos, com nenhum sinal clínico de infecção pelo protozoário Leishmania; oligossintomáticos, apresentando linfadenopatia, ligeira perda de peso e/ou alopecia; ou sintomáticos, mostrando todos ou alguns dos vários sintomas da doença, como lesões cutâneas, onicogrifose, ceratoconjuntivite e rigidez dos membros posteriores.

$\mathrm{Na}$ LVC, a distribuição do parasito pode ser extensa, alcançando principalmente órgãos como baço, fígado, linfonodos, pele, medula óssea ${ }^{24}$. São também descritos acometimentos do sistema nervoso central, rins, pâncreas, testículo, olho, glândula lacrimal, glândula adrenal, placenta e trato gastrintestinal (estômago, duodeno, jejuno, íleo, ceco e cólon) ${ }^{49,50,51,52,53,54,56,57}$.

A pele íntegra apresenta intenso parasitismo em cães com LV. Locais como focinho, pavilhão auricular, abdômen e escápula são citados como principais sítios de intenso parasitismo de pele sã $^{58,59}$. Entretanto, ainda não há definição do sitio de eleição para o diagnóstico dentre estes sítios citados acima. 
Os linfonodos cervicais e poplíteos apresentam grande parasitismo. De acordo com Maderia et al. (10) amostras biológicas de linfonodos cervicais apresentam maior positividade, quando comparado com amostras biológicas de linfonodos poplíteos. Entretanto, Lima et al. ${ }^{(60)}$ não observou diferença de parasitismo entre amostras biológicas de linfonodos cervicais e poplíteos.

Pela gravidade da LV, as ações sanitárias direcionadas a essa doença são mais rigorosas que aquelas direcionadas a LTA. A estratégia de controle da LV se resume em três medidas principais: 1) detecção e tratamento dos casos humanos, 2) borrifação no domicílio e peridomicílio com inseticidas apropriados, associada ao monitoramento da fauna flebotomínica e 3) identificação e eliminação de cães sorologicamente positivos ${ }^{31}$.

A estratégia da eutanásia de cães sorologicamente positivos é um assunto polêmico. De acordo com Costa et al. (1999) ${ }^{61}$ existe um risco duas vezes maior de contrair a infecção em residências onde vivem cães. Braga et al. $(1998)^{62}$, constatou significativa redução no surgimento de novos casos quando cães infectados foram rapidamente retirados da área. No entanto, outros estudos sugerem que a eliminação de cães soro positivos exerce pouca ou nenhuma influência no surgimento de novos casos humanos ou caninos ${ }^{42,63}$. Nesse contexto, apesar da participação o cão doméstico (Canis familiaris), como reservatório ser conhecida, busca-se justificar com segurança a prática da eutanásia canina como uma das medidas de controle.

\section{LEISHMANIOSE TEGUMENTAR}

A LTA é uma doença infecto-parasitária, não contagiosa, restrita ao tegumento, amplamente distribuída no território brasileiro. Considera-se que 1,5 milhões de novos casos de Leishmaniose tegumentar americana (LTA) humana ocorram anualmente no mundo. Também conhecida como leishmaniose mucocutânea, úlcera de Bauru, ferida brava entre outros, a LTA, apresenta ampla distribuição nas Américas Central e do Sul. No Brasil, tem sido relatada nas regiões Norte, Nordeste, Centro-Oeste, Sul e Sudeste ${ }^{64}$ e é causada pelas espécies Leishmania (Viannia) braziliensis, Leishmania (Leishmania) amazonensis, Leishmania (Viannia) guyanensis, Leishmania (Viannia) lainsoni, Leishmania (Viannia) shawi, Leishmania (Viannia) naiffi ${ }^{65}$.

A leishmaniose tegumentar canina (LTC) é uma doença de evolução crônica cujas manifestações clínicas restringem-se à pele e às mucosas das viasaero-digestivas superiores. A úlcera cutânea típica costuma ser única, eventualmente múltipla, localizada nas orelhas, bolsa escrotal e focinho ${ }^{66}$.

A LTC tem sido registrada em várias regiões do Brasil, como Rio de Janeiro, São Paulo, Espírito. Santo, Minas Gerais, Bahia e Amazonas ${ }^{67}$.

A importância dos cães na transmissão da LT ainda não está esclarecida, alguns autores os consideram como reservatórios secundários da doença ou elo na cadeia de transmissão humana ${ }^{68}$, 69,70 , e outros sugerem que o cão se infecta acidentalmente, assim como o homem ${ }^{71,72,73}$.

\section{DIAGNÓSTICO DAS LEISHMANIOSES CANINAS}


O diagnóstico das leishmanioses caninas baseia-se nos sinais clínicos, epidemiologia, demonstração do parasito por diferentes técnicas e testes sorológicos. Para a confirmação do diagnóstico, é necessária a demonstração do agente etiológico nas amostras biológicas obtidas das lesões cutâneas ou mucosas ulceradas, na LTC $3,{ }^{74}$, aspirado de fígado, baço, medula óssea, lesões cutêneas, pele íntegra ou de gânglios linfáticos na $\mathrm{LVC}^{10,63}$.

A observação das formas amastigotas de Leishmaniasp. pode ser realizada de forma direta, por meio da citopatologia, histopatologia, isolamento em cultura e inoculação emhamsters, ou indiretamente, por meio de técnicas imuno-histoquímicas, hibridização com sondas de DNA e reação em cadeia de polimerase (PCR)75. A confirmação parasitológica e etiológica de cães suspeitos é de fundamental importância, não só para o conhecimento das espécies circulantes nesses animais, mas também na contribuição de elementos que possam ser úteis nas ações de controle.

As coletas de amostras biológicas por meio do aspirado de fígado, baço e medula óssea, são técnicas invasivas, o que dificulta a obtenção destas amostras de cães, principalmente, em visitas domiciliares. Entretanto, amostras biológicas de linfonodos e de pele íntegra são coletadas por técnicas menos invasivas, o que facilita a coleta de amostras biológicas em cães, principalmente em visitas domiciliares.

Os testes sorológicos teste rápido DPP-LVC e ensaio imunoenzimático (ELISA) são os mais utilizados em estudos epidemiológicos e inquéritos para medidas de controle da LVC, devido à facilidade da coleta de amostras, baixos custos, rapidez do diagnóstico e a devido à resposta imune humoral que ocorre nos cães com LV que facilita o diagnóstico sorológico ${ }^{31,76}$. Alguns autores questionam o valor destes testes sorológicos no diagnóstico das leishmanioses caninas pela existência de reação cruzada com outras enfermidades, como doença de Chagas e esporotricose ${ }^{77,78}$ pela impossibilidade de identificação do parasito ${ }^{79}$ e pela ocorrência de cães soro negativos que apresentavam cultura parasitológica positiva ${ }^{6}$.

\section{LEISHMANIOSE EM BRASÍLIA}

O impacto das leishmanioses na saúde pública esteve extremante subestimado durante muitos anos e considera-se que 2 milhões de casos novos (1,5 milhões para LT e 500.000 para LV) ocorram anualmente, com uma estimativa de 12 milhões de pessoas infectadas atualmente em todo o mundo ${ }^{1}$.

A importância das leishmanioses em saúde pública tem aumentado, não só pela expansão geográfica, mas também pela sua introdução em áreas anteriormente indenes e pela instalação de focos de transmissão em áreas urbanizadas.

Em 2012, o Brasil registrou 1171 casos humanos, confirmados com diagnóstico parasitológico de leishmaniose visceral, sendo 80 casos na região norte, 649 casos na região nordeste, 
305 casos na região sudeste, 134 casos na região centro-oeste e 3 casos na região sul. No Distrito Federal, houve 13 casos (sendo 7 casos autóctones), dos quais 1 caso evoluiu para óbito ${ }^{80}$.

No Distrito Federal, de acordo com Sistema de Informação de Agravos e Notificação - SINAN, foram diagnosticados 73 casos de LV humana no período de 2004 a 2013, e 518 casos de LTA humana no período de 2001 a 2013. Casos de leishmaniose canina foram diagnosticados por meio de testes sorológicos nas seguintes regiões administrativas: Águas Claras, Brasília, Brazlândia, Candangolândia, Ceilândia, Estrutural, Fercal, Paranoá, Park Way, Guará, Guará II, Itapoã, Jardim Botânico, Lago Norte, Lago Sul, Núcleo Bandeirante, Planaltina, Recanto das Emas, Riacho Fundo I, Riacho Fundo II, Samambaia, Santa Maria, São Sebastião, SIA, Sobradinho, Sobradinho II, Taguatinga,Varjão ${ }^{81}$. Dentre estas regiões administrativas as que apresentam maiores incidências de leishmaniose canina no ano de 2013 foram Fercal, Lago Norte, Jardim Botânico e Sobradinho ${ }^{81}$.

O Programa Nacional de Controle da Leishmaniose Visceral (PVC-LV) baseia-se no tratamento dos casos humanos, na detecção e eliminação dos cães soropositivos, no combate ao vetor, no manejo ambiental e na vigilância epidemiológica. Entretanto, enfatiza a necessidade da realização das ações de forma integrada, e reforça a inefetividade das mesmas em reduzir a incidência de LV quando realizadas de forma isolada.

A eutanásia do reservatório urbano é o item mais criticado do programa e enfrenta limitações como o longo intervalo entre o diagnóstico e a remoção do cão; sensibilidade e especificidade dos exames menor que a ideal; a não aceitação dos proprietários em submeter seus cães à eliminação; e a rápida reposição canina ${ }^{63,82}$.

Dados da Fundação Nacional de Saúde apontam que de 1980 a 1991 foram coletadas amostras de sangue de 4.030 .762 cães brasileiros e realizados 3.719 .965 exames, dos quais 111.791 foram positivos (3,01\%). Nesse período, 137.243 animais infectados ou não domiciliados foram eliminados e houve redução da soroprevalência canina, a qual foi creditada à eficiência das ações de controle83. Segundo Dietze et al., (1997)84, entre 1990-1994, foram testados no Brasil mais de 4,5 milhões de cães, dos quais 80.000 foram eliminados, contudo, a incidência de casos humanos da doença no mesmo período apresentou $100 \%$ de aumento.

Fatores determinantes do adoecimento por LV não se restringem ao campo biológico, existe a necessidade de se implantar políticas públicas destinadas a corrigir desigualdades socioeconômicas que restringem o acesso a direitos e serviços básicos, como saúde, moradia, alimentação, renda e saneamento $^{83}$. Entretanto, em Brasília temos casos de leishmaniose canina em locais de classe de alta renda como o Lago Norte e Lago Sul, onde as condições socioeconômicas permitem o acesso à saúde e saneamento ${ }^{81}$.

Cães com LV apresentam expressiva resposta imune humoral, devido a isto, inquéritos soro epidemiológicos são realizados visando o rastreamento de cães supostamente infectados. Ainda que o 
diagnóstico de rotina da LVC se baseie em dados sorológicos, para a confirmação da infecção é necessária a demonstração direta ou indireta do parasita por métodos parasitológicos, uma vez que a sorologia não possui poder discriminatório entre a forma tegumentar e visceral, e a garantia da circulação de uma dada espécie somente será possível após a identificação do agente etiológico.

Entretanto, em Brasília - DF, apesar da eutanásia canina de cães sororeatores serem realizada, testes confirmatórios da infecção não são realizados sendo poucos os estudos que mencionam os agentes circulantes nesses animais ${ }^{81}$.

São muitos os questionamentos e poucas as respostas que fazem da leishmaniose um assunto bastante debatido na sociedade, o que demonstra a necessidade imediata de se produzir conhecimento fundamentado sobre o agente, vetor, reservatório e hospedeiro, bem como sobre os determinantes históricos, econômicos e sociais da infecção e adoecimento, uma vez que a moléstia, complexa e multifatorial, não pode ser analisada somente sob a óptica biológica.

Desta forma, considera-se pertinente realização de pesquisas, que contribuam para o aprimoramento das estratégias de prevenção e controle da leishmaniose canina no Distrito Federal.

\section{CONCLUSÃO}

Apesar das ações de vigilância e controle de LV adotadas em Brasília (DF), a doença permanece em áreas urbanas e rurais, em locais de boas condições socioeconômicas onde a população tem acesso à saúde e saneamento, e em locais de precárias condições socioeconômicas, enfatizando a carência de estudos que assinalem medidas mais efetivas para eliminar os vetores e reduzir a prevalência de LVC.

\section{REFERÊNCIAS BIBLIOGRÁFICAS}

1. World Health Organization (WHO). Health topics: Leishmaniasis. Disponível em: $<$ http// www.who.int/em> (Acesso em 20. set.2015).

2.Lainson R, Shaw J. Evolution, classification and geografical distribution. In: Peteres, KillickKendrick., editors. The leishmaniasis in biology and medicine. London: Academic press; 1987.

3. Marzochi MAC, Marzochi KBF. Tegumentary and visceral leishmaniases in Brazil. Emerging anthropozoonosis and possibilities for their control. Cad. SaúdePública 1994; 10:359375.

4. Marzochi MAC. Leishmanioses no Brasil: As leishmanioses tegumentares. BrasMed. 1992; 63(82-104).

$196 / /$

5.Dantas-Torres F. Canine leishmaniosis in South America.ParasitVectors. 2009; 26;2 Suppl 1:S1. 
6.Madeira MF, Schubach A, Schubach TM, Pacheco RS, Oliveira FS, Pereira SA et al. Mixed infection with Leishmania (Viannia) braziliensis and Leishmania (Leishmania) chagasi in a naturally infected dog from Rio de Janeiro, Brazil. Trans R Soc Trop Med Hyg.2006; 100(5):442-

5.

7.Deane LM, Deane MP, Alencar JE. Control of Phlebotomuslongipalpis by DDT house spraying endemic foci of kala-azar in Ceara. Rev Bras Malariol Doencas Trop. 1955; 7(1):131-41.

8.Marzochi MC, Coutinho SG, Souza WJ, Toledo LM, Grimaldi Junior G, Momen H et al. Canine visceral leishmaniasis in Rio de Janeiro, Brazil. Clinical, parasitological, therapeutical and epidemiological findings (1977-1983). Mem. Inst. Oswaldo Cruz. 1985; 80(3):349-357.

9.Madeira MF, Schubach AO, Schubach TMP, Leal CA, Marzochi MCA Identification of Leishmania(Leishmania) chagasiIsolated from Healthy Skin Symptomatic and Asymptomatic Dogs Soropositive for Leishmaniasis in the Municipality of Rio de Janeiro, Brazil. Braz J Infect.2004; 8: 440-444.

10.Madeira MF, Schubach AO, Schubach TMP, Pereira SA, Figueiredo FB, Baptista C et al. Post mortem parasitological evaluation of dogs seroreactive for Leishmania from Rio de Janeiro, Brazil. Vet. Parasitol. 2006B; 138:366-370.

11. Xavier SC, Andrade HM, Hadad S, Chiarelli I, Lima WG, Michalick MSM et al. Comparation of paraffin-embedded skin biopsies from different anatomical regions as sampling methods for detecting Leishmania infection in dogs using histological, immunohistochemical and PCR methods. BMC Vet Res. 2006; 2(17):1746-1756.

12. Verçosa BL, Lemos CM, Mendonça IL, Silva SM, de Carvalho SM, Goto H et al. Transmission potential, skin inflammatory response, and parasitism of symptomatic and asymptomatic dogs with visceral leishmaniasisBMC Vet Res.2008; 6;4:45.

13. Madeira MF, Figueiredo FB, Pinto AG, Nascimento LD, Furtado M, Mouta-Confort E et al. Parasitological diagnosis of canine visceral leishmaniasis: is intact skin a good target? Res VetSci. 2009; 87(2):260-2.

14.Calabrese KS, Cortada VM, Dorval ME, Souza Lima MA, Oshiro ET, Souza CS, SilvaAlmeida $\mathrm{M}$ et al. Leishmania (Leishmania) infantum/chagasi: Histopathological aspects of the skin in naturally infected dogs in two endemic areas.ExpParasitol.2010; 124(3):253-7. Epub 2009 Oct 23.

15.Mauricio IL, Howard MK, Stothard JR, Miles MA. Genomic diversity in the Leishmaniadonovani complex. Parasitology. 1999; 119 (3):237-46. 
16.Marcondes M, Biondo AW, Gomes AA, Silva AR, Vieira RF, Camacho AA et al. Validation of a Leishmaniainfantum ELISA rapid test for serological diagnosis of Leishmaniachagasi in dogs.Vet Parasitol. 2011; 10;175(1-2):15-9.

17.Desjeux P. Leishmaniasis. Public health aspects and control. ClinDermatol 1996 SepOct;14(5):417-23.

18.Chappuis F, Sundar S, Hailu A, Ghalib H, Rijal S, Peeling RW et al. Visceral leishmaniasis: What are the needs for diagnosis, treatment and control? Nat VerMicrobiol. 2007; 5(11):873-82.

19 Ready PD. Leishmaniasis emergence in Europe. Euro Surveill. 2010; 15(10):p.195-205.

20.Petersen C.A., Leishmaniasis, an Emerging Disease Found in Companion Animals in the United States Topics. Companion Animal Medicine Volume 24, Number 4, November 2009.

21.Shaw JJ. Taxonomy of the genus Leishmania: present and future trends and their implications. Mem Inst Oswaldo Cruz. 1994; 89(3):471-8.

22.Shaw JJ. Further thoughts on the use of the name Leishmania (Leishmania) chagasifor the aetiological agent of American Visceral Leishmaniasis. Mem Inst Oswaldo Cruz2006; 101(5):577-579.

23. Slappendel RJ, Ferrer L. Leishmaniasis. In: Greene CE. Enfermidades infecciosas enperros y gatos. 2 ed. México: Interamericana McGraw-Hill; 2000; 496-504.

24. Alvar J, Cañavate C, Molina R, Moreno J, Nieto J. Canine Leishmaniasis. Adv Parastol 2004; 57:1-88.

25. Pocai EA, Frozza L, Headley SA, Graça DL. Leishmaniose visceral (calazar). Cinco casos em cães de Santa Maria, Rio Grande do Sul, Brasil. Cienc Rural.1998; 28: 501-505.

26. Monteiro PS. Leishmaniose visceral no Brasil: Perspectivas de controle. Rev Soc Bras Med Trop. 2002; 35(1):335.Monteiro, 2002;

27.Souza GD, Santos E, Andrade Filho JD. The first report of the main vector of visceral leishmaniasis in America, Lutzomyialongipalpis (Lutz \& Neiva) (Diptera: Psychodidae: Phlebotominae), in the state of Rio Grande do Sul, Brazil. Mem Inst Oswaldo Cruz.2009; 104(8):1181-2.

28.Grimaldi G Jr, Tesh RB, McMahon-Pratt D. A review of the geographic distribution and epidemiology of leishmaniasis in the New World. Am J TropMedHyg 1989 Dec;41(6):687-725.

29. Vieira JBF, Coelho GE. Leishmaniose visceral ou calazar: aspectos epidemiológicos e de controle. RevSocBrasMed Trop. 1998; 31(2):85-92. 
30. Marzochi MC, Fagundes A, Andrade MV, Souza MB, Madeira Mde F, Mouta-Confort E et al. Visceral leishmaniasis in Rio de Janeiro, Brazil: eco-epidemiologicalaspectsandcontrol. RevSocBrasMedTrop.. 2009; 2(5):570-80.

32.Silva ES, Gontijo CM, Pacheco RS, Fiuza VO, Brazil RP. Visceral leishmaniasis in the Metropolitan Region of Belo Horizonte, State of Minas Gerais. Brazil. Mem Inst Oswaldo Cruz. 2001; 96(3):285-91.

33. Barata RA, França-Silva JC, Costa RT, Fortes-Dias CL, Silva JC, Paula EV et al. Phlebotomine sand flies in Porteirinha, an area of American visceral leishmaniasis transmission in the State of Minas Gerais, Brazil. Mem Inst Oswaldo Cruz, 2004; 99(5):481-7.

34. Michalsky EM, Rocha MF, Lima ACVM, França-Silva R, Pires MQ, Oliveira FS et al. Infectivity of soropositive dogs, showing differente clinical forms of leishmaniasis, to Lutzomyialongipalpisphlebotomine sand flies. Vet Parasitol 2007; 20;147(1-2):67-76.

35. Souza GD, Santos E, Andrade Filho JD. The first report of the main vector of visceral leishmaniasis in America, Lutzomyialongipalpis (Lutz \& Neiva) (Diptera: Psychodidae: Phlebotominae), in the state of Rio Grande do Sul, Brazil. Mem Inst Oswaldo Cruz. 2009; 104(8):1181-2.

36. Genaro O, da Costa CA, Williams P, Silva JE, Rocha NM, Lima SL et al. Occurrence of kala-azar in the urban area of Grande Belo Horizonte, Minas Gerais. Rer. Soc. Bras. Med. Trop. 1990; 23(2):121.

37. Santa Rosa ICA, Oliveira ICS. Leishmaniose visceral: breve revisão sobre uma zoonose reemergente. Clinica Veterinária. 1997; (2):24-28.

38. Palatnik-de-Sousa CB, dos Santos WR, Franca-Silva JC, da Costa RT, Reis AB, Palatnik $M$ et al. Impact of canine control on the epidemiology of canine and human visceral leishmaniasis in Brazil. Am J Trop Med Hyg. 2001; 65(5):510-7.

39. Chagas E, Cunha AM, Ferreira LC, Deane L, Deane G, Paumgartteen MP. Leishmaniose visceral americana (relatório dos trabalhos pela Comissão encarregada do estudo da leishmaniose visceral americana em 1937. Mem Inst Oswaldo Cruz 1938; 33: 89-229.

40. Alencar JE, Cunha RV. Inquérito sobre o calazar no Ceará. Novos resultados. Rev Bras Malariol Doenças Trop. 1963; 15:391-403.Alencar \& Cunha, 1963.

41. Alencar JE. Contribuição para o estudo da epidemiologia no Brasil: Calazar canino. 1959. Imprensa Oficial, Fortaleza, 342p.

42. Paranhos-Silva M, Nascimento EG, Melro MC, Oliveira GG, Santos WL, Pontes-deCarvalho LC et al. Cohort study on canine emigration and Leishmaniainfection in an endemic 
area for American visceral leishmaniasis. Implications for the diseasecontrol. Acta Trop. 1998; 69(1):75-83.

43. Galimbertti, M. Z., Katz G. Leishmaniose visceral americana no Estado de São Paulo. Rev Soc Bras Med Trop. 1999; (1):217.

44. Cabrera MA, Paula AA, Camacho LA, Marzochi MC, Xavier SC, da Silva AV et al. Canine visceral leishmaniasis in Barra de Guaratiba, Rio de Janeiro, Brazil: assessment of risk factors. Rev Inst Med Trop Sao Paulo. 2003; 45(2):79-83.

45 Marzochi MCA, Marzochi KBF. Leishmaniosesem áreas urbanas. Rev Soc Bras Med Trop. 1997; 30:162-4.

46 Almeida, MA., Jesus EE. Clinical and serological aspects of visceral leishmaniasis in northeast Brazilian dogs naturally infected with Leishmaniachagasi. VetParasitol. 2005; (127):227-32.

47 Blavier A, Keroack S, Denerolle P, Goy-Thollot I, Chabanne L, Cadore JL et al. Atypical forms of canine leishmaniosis. Vet J. 2001; 162(2):108-20.

48Mancianti F, Gramiccia M, Gradoni L, Pieri S. Studies on canine leishmaniasis control.1. Evolution of infection of different clinical forms of canine leishmaniasis following antimonial treatment. Trans R Soc Trop Med Hyg. 1988; 82(4):566-7.

49. Ferrer L, Rabanal RM, Domingo M, Ramos JA, Fondevila D. Identification of Leishmaniadonovani amastigotes in canine tissues by immunoperoxidase staining. Res. Vet. Sci. 1988; 44(2):194-196.

50. Tafuri WL, Tafuri WL, Barbosa AJ, Michalick MS, Genaro O, França-Silva JC et al. Histopathology and immunocytochemical study of type 3 and type 4 complement receptors in the liver and spleen of dogs naturally and experimentally infected with Leishmania (Leishmania) chagasi. Rer. Inst. Med. Trop. S. Paulo 1996; 38(2):81-89.

51. Bourdoiseau G, Marchal T, Magnol JP. Immunohistochemical detection of Leishmaniainfantum in formalin-fixed, paraffin-embedded sections of canine skin and lymph nodes. J. Vet. Diagn. Invest. 1997; 9(4):439-440.

53. Tafuri WL, Santos Rde L, Arantes RM, Goncalves R, de Melo MN, Michalick MS. An alternative immunohistochemical method for detecting Leishmania amastigotes in paraffin-embedded canine tissues. J. Immunol. Methods. 2004; 292(1-2):17-23.

54. Ordeix L, Solano-Galeno L, Fondevila D, Ferrer L, Fondat A. Papular dermatitis due to Leishamniaspp. infection in dogs with parasite-specific cellular immune responses. Vet. Dermatol. 2005; 16:187-191 
55. Naranjo C, Fondevila D, Leiva M, Roura X, Pena T. Characterization of lacrimal gland lesions and possible pathogenic mechanisms of keratoconjunctivitis sicca in dogs with leishmaniosis. Vet. Parasitol. 2005; 133(1):37-47.

56.Dubey J, Rosypal A, Pierce V, Scheinberg S, Lindsay D. Placentitis associated with leishmaniasis in a dog. J. Am. Vet. Med. Assoc. 2005; 227(8):1266-1269.

57. Okamoto, T. Avaliação Clínica e anatomopatológica das leishmanioses caninas no Estado do Rio de Janeiro. Dissertação de mestrado (Mestrado em medicina veterinária coletiva) Universidade Federal Fluminense, 2006.

58. Madeira MF, Schubach AO, Schubach TMP, Serra CM, Pereira SA, Figueiredo FB eT al. Is Leishmania (Viannia) braziliensispreferentially restricted to the cutaneous lesions of naturally infected dogs? Parasitol Res. 2005; 97(1):73-6.

59. Calabrese KS, Cortada VM, Dorval ME, Souza Lima MA, Oshiro ET, Souza CS, SilvaAlmeida $\mathrm{M}$ et al. Leishmania (Leishmania) infantum/chagasi: Histopathological aspects of the skin in naturally infected dogs in two endemic areas.ExpParasitol. 2010; 124(3):253-7. Epub 2009 Oct 23.

60. Lima WG, Michalick MSM, Melo MN, Tafuri WL. Canine visceral leishmaniasis: a histopathological study of lymph nodes. Acta Trop. 2004; 92: 43-53.

61. Costa CH, Pereira HF, Pereira FC, Tavares JP, Araujo MV, Goncalves MJ. Is the household dog a risk factor for American visceral leishmaniasis in Brazil? Trans R Soc Trop Med Hyg. 1999; 93(5):464.

62.Braga MD, Coelho IC, Pompeu MM, Evans TG, MacAullife IT, Teixeira MJ et al. Control of canine visceral leishmaniasis: comparison of results from a rapid elimination program of serumreactive dogs using an immunoenzyme assay and slower elimination of serum-reactive dogs using filter paper elution indirect immunofluorescence. RevSocBrasMed Trop. 1998; 31(5):419-24.

64 Ministério da Saúde SdVeS, Departamento de Vigilância Epidemiológica. Manual de vigilância e controle da leishmaniose tegumentar americana. $2^{\mathrm{a}}$ ed. Brasília: Ministério da Saúde; 2007.

65. Genaro O. Leishmaniose tegumentar americana. In: Neves DP, editor. Parasitologia humana. São Paulo: Atheneu; 1995.

66. Madeira MF, Uchôa CM, Leal CA, Silva RM, Duarte R, Magalhães CM et al. Leishmania (Viannia) braziliensis in naturally infected dogs. Rev. Soc. Bras. Med. Trop. 2003; 36(5):551555. 
67. Tafuri W.L., RASO P., Hermeto M.V., Vieira-Dias D. \& Mayrink W. 1993. Comparative histopathologic study of the skin test in dogs from an endemic area of tegumentaryleishmaniasis, using 2 antigens: Leishvacin and P10.000G. Rev. Soc. Bras. Med. Trop. 26:11-4. $201 / /$

68 Dias M., Mayrink W., Deane L.M., Da Costa C.A., Magalaes P.A., Melo M.N., Batista S.M., Araujo F.G., Coelho M.V. \& Willian P. 1977. Epidemiology of mucocutanoeusleishmaniasis Americana. I. Study of reservoirs in an endemic region of the State of Minas Gerais. Ver. Inst. Med. Trop. São. Paulo. 19:403-10.

69. Coutinho S.G., Nunes M.P., Marzochi M.C.A. \&Tramontano N. 1985. A survey for american cutaneous and visceral leishmaniasis among 1,342 dogs from áreas in Rio de Janeiro (Brazil) where the human deseases occur. Mem. Inst. Oswaldo Cruz. 80:17-22.

70 Falqueto A., Coura J.R., Barros G.C., Grimaldi-filho G., Sessa P.A., Carias V.R., De Jesus A.C. \& D Alencar J.T. 1986. Participation of the dog in the cycle of transmission of cutaneous leishmaniasis in the municipality of Viana, State of Espirito Santo, Brazil. Mem. Inst. Oswaldo Cruz. 81:155-63.

71 Fortani PO. Sobre os reservatórios naturais da leishmaniose tegumentar americana. Rev. Inst. Med. Trop. S. Paulo 1960; 2:195-203.

72. Reithinger R, Davies CR. Is the domestic dog (Canisfamiliaris) a reservoir host of American cutaneous leishmaniasis? A critical review of the current evidence. Am. J. Trop. Med. Hyg. 1999; 61(4):530-41.

73. Savani E.S., Galati E.A., Camargo M.C., D’auria S.R., Damaceno J.T. \&Balduino S.A. 1999. Serological survey for American cutaneous leishmaniasis in stray dogs in the S. Paulo State, Brazil. Rev. Saúde Pública. 33:629-31.

74. Dos Santos IB, Tortelly R, Quintella LP, Fátima Madeira M,Monteiro de Miranda LH, Borges Figueiredo F, Carvalhaes de Oliveira Rde V, Pacheco Schubach TM. Higher sensitivity of immunohistochemistry for bona fide diagnosis of dog Leishmania (Viannia) braziliensis-driven American tegumentaryleishmaniasis: description of an optimized immunohistochemistry method. Trans R SocTropMed Hyg.2015 Jul;109(7):469-76.

75 Da Silva RN, Amorim AC, Brandão RM, de Andrade HM, Yokoo M, Ribeiro ML et al. Real-time PCR in clinical practice: a powerful tool for evaluating Leishmaniachagasi loads in naturally infected dogs. TropMedParasitol. 2010; 104(2):137-43.

76. Nota Técnica Conjunta nº 01/2011-CGDT-CGLAB/DEVIT/ SVS/MS.

Esclarecimentos sobre substituição do protocolo diagnóstico da leishmaniose visceral canina (LVC). CoordenaçãoGeral de DoençasTransmissíveis/CoordenaçãoGeral de Laboratórios de Saúde Pública. Brasília: 29 de dezembro de 2011. 
77 Reed SG, Shreffler WG, Burns JM, Jr., Scott JM, OrgeMda G, Ghalib HW et al. An improved serodiagnostic procedure for visceral leishmaniasis. Am. J. Trop. Med. Hyg. 1990; 43(6):632-639. $202 / /$

78. Santos IB, Schubach TMP, Paes-Leme LR, Okamoto T, Figueiredo FB, Pereira SA et al. Sporotrichosis - The main differential diagnosis with tegumentary leishmaniosis in dogs from Rio de Janeiro, Brazil. Vet. Parasitol. 2007; 143(1):1-6.

79. De Paula A A, Da Silva AV.The use of immunoblot analysis in the diagnosis of canine visceral leishmaniasis in an endemic area of Rio de Janeiro. J Parasitol. 2003; (89):832-6.

80 SINAN - Sistema de Informação de Agravos de Notificação. Disponívelem: http://dtr2004. saude.gov.br/sinanweb/ (acessadoem 20-set-2015)

81 Herenio, E.M, Fortes, R.C; Rincon, G. Prevalência da Leishmaniose visceral em cães do Distrito Federal, segundo dados do centro de zoonoses de Brasília Prevalenceof visceral Leishmaniasis in dogsof Federal District, according to center of zoonoses Brasília. J Health Sci Inst. 2014;32(2):126-9

82, Moreira MA, Luvizotto MC, Garcia JF, Corbett CE, Laurenti MD. Comparison of parasitological, immunological and molecular methods for the diagnosis of leishmaniasis in dogs with different clinical signs. Vet Parasitol.2007; 30;145(3-4):245-52.

83. Lacerda M. The Brazilian leishmaniasis control program. Mem. Inst. Oswaldo Cruz 1994; 89(3):489-495.

84 Deyize J, Rosypal A, Pierce V, Scheinberg S, Lindsay D. Placentitis associated with leishmaniasis in a dog. J. Am. Vet. Med. Assoc. 1997; 227(8):1266-1269.

Artigo apresentado em 10-10-14

Artigo aprovado em 12-04-15 Artigo publicado no sistema em 22-12-15 